\title{
COMUNICAÇÃO
}

\section{COMPARAÇÃO DE AMBIENTES NA GERMINAÇÃO DE CARIOPSES DE CANA-DE-AÇÚCAR}

\author{
Environment comparison in the germination of sugarcane seeds
}

\author{
Marcelo de Almeida Silva1 ${ }^{1}$, Marina Maitto Caputo², Dilermando Perecin ${ }^{3}$ José Antônio Bressiani ${ }^{4}$
}

\begin{abstract}
RESUMO
A obtenção de cariopses de cana-de-açúcar por meio de cruzamento de parentais é de primordial importância para os programas de melhoramento de cultivares. Objetivou-se, neste trabalho comparar o ambiente de germinação de cariopses da casa de vegetação do Programa Cana IAC com um ambiente de condições controladas, a fim de avaliar sua eficiência, no período de 30/09 a 09/ 10/2004. Foram desenvolvidos dois experimentos, em delineamento experimental de blocos casualizados com quatro repetições, em Jaú, SP. Um deles foi montado sob condições de laboratório em câmara de germinação e outro em casa de vegetação em condições não controladas, sendo cada tratamento constituído por 100 cariopses por repetição, provenientes de seis cruzamentos. Foram realizadas avaliações do índice de velocidade de germinação (IVG), da porcentagem (\%G) e dos dias para emergência (DE), em intervalos de 24 horas, após a instalação dos testes. A massa de 100 sementes de cada cruzamento também foi avaliada com o propósito de verificar sua influência nos demais atributos da germinação. Os resultados foram submetidos à análise de variância e a comparação de médias foi feita pelo teste de Tukey, a 0,05 de significância. A massa de 100 sementes foi diferente entre os cruzamentos, e interferiu, de maneira geral, nos atributos IVG e \% G. Concluiu-se que a casa de vegetação utilizada pelo Programa Cana IAC proporciona porcentagens de germinação semelhantes às da câmara de germinação, porém condições controladas favorecem o IVG e os DE.
\end{abstract}

Termos para indexação: Saccharum sp., vigor de plântulas e índice de velocidade de germinação.

\begin{abstract}
The production of true seeds of sugarcane through parental crossing is primordial for the breeding programs of cultivars. With the objective of comparing the efficiency of the environment greenhouse used for seed germination in the IAC Sugarcane Program with an environment with controlled conditions (germination chamber), two experiments were carried out in a completely randomized block with four replicates of 100 seeds each, in Jaú (SP), from 09/30 to 10/09/2004. In both places the treatments were composed of seeds from six crossings. The evaluations accomplished were speed of germination index (SGI), germination percentage (\% G) and days for emergence (DE) in $24 \mathrm{~h}$ intervals after set up treatments. The mass of 100 seeds of each crossing was also evaluated with the purpose of measuring its influence on other germination attributes. The data were submitted to the analysis of variance and averages were compared by the Tukey test at 0.005 significance. The weight of 100 seeds differed among the crossings, and, in general, it interfered with the attributes SGI and \% G. It was concluded that the greenhouse used by the IAC Sugarcane Program provides germination percentages similar to the germination chamber; however, controlled conditions improve SGI and DE.
\end{abstract}

Index terms: Saccharum sp., seedling vigor and speed of germination index.

(Recebido em 2 de agosto de 2007 e aprovado em 25 de março de 2008)

A obtenção de cariopses de cana-de-açúcar por cruzamento de parentais é de primordial importância para os programas de melhoramento de cultivares, uma vez que elas dão suporte aos processos de seleção e desenvolvimento de novas cultivares.

Até meados do século 19, os botânicos não haviam detectado que a cana-de-açúcar produzia cariopses. Segundo Machado Júnior (1987), naquela época pensavase que a propagação assexual contínua havia causado à cana-de-açúcar a perda da capacidade de reprodução sexual. Em 1842 houve o primeiro relato, feito pelo brasileiro Gervásio Caetano Peixoto Lima, de que a cana-de-açúcar se reproduzia sexuadamente. Em 1887, produziram-se as primeiras cariopses, de cruzamentos dirigidos entre Saccharum spontaneum e Saccharum officinarum, na ilha de Java, constituindo o primeiro programa de hibridação em canade-açúcar, seguido posteriormente por Barbados, em 1889 e o Havaí, em 1905 (Miocque \& Machado Júnior, 1977).

\footnotetext{
${ }^{1}$ Agência Paulista de Tecnologia dos Agronegócios/APTA - Regional Centro Oeste - Rodovia Deputado Leônidas Pacheco Ferreira - Km 304 Cx. P. 66 - 17201-970 - Jaú, SP - marcelosilva@apta.sp.gov.br

¿Universidade de São Paulo/USP - Escola Superior de Agricultura "Luiz de Queiroz"/ESALQ - Departamento de Produção Vegetal - Piracicaba, SP

3Universidade Estadual Paulista Júlio de Mesquita Filho/ UNESP - Departamento de Ciências Exatas - Jaboticabal, SP

${ }^{4}$ Empresa Canavialis S.A. - Campinas, SP
}

Ciênc. agrotec., Lavras, v. 34, Edição Especial, p. 1604-1609, 2010 
Desde então alguns programas de melhoramento têm problemas para a germinação de cariopses e a formação das plântulas. Os maiores problemas são: a) perda da viabilidade de cariopses durante a estocagem e a manipulação; b) instalações e materiais deficientes para germinação, e c) perda de mudas nos primeiros estádios de crescimento (Breaux \& Miller, 1987), uma vez que as plântulas de canade-açúcar são muito delicadas e vulneráveis nas quatro primeiras semanas depois da germinação.

Segundo Breaux \& Miller (1987), são condições fundamentais para a germinação da semente e o estabelecimento das plântulas: a) temperatura e água; b) um substrato bem drenado; c) equilíbrio dos nutrientes, e c) controle das doenças. Em condições ótimas disponibilidade de água e de temperatura, os primeiros sinais de germinação, como mudança de cor no tegumento, podem ser percebidos em 24 horas; após 48 horas, as raízes primárias rompem o tegumento e no terceiro dia o coleóptilo, no sétimo os primórdios foliares iniciam o crescimento e aos 10 dias a segunda folha emerge (Dillewijn, 1952; Lucchesi, 2001).

Heinz (1975) indicou que, para cinco cultivares de cana-de-açúcar provenientes do Havaí, a maior taxa de germinação das cariopses e o maior vigor de mudas foram obtidos a $38^{\circ} \mathrm{C}$. A manutenção da temperatura constante, entre 30 e $35^{\circ} \mathrm{C}$ é, provavelmente, o fator mais importante para a germinação de cariopses da cana-de-açúcar e o crescimento de suas mudas. Em regiões subtropicais, onde temperaturas mais frias podem ocorrer durante os meses de inverno, estufas completamente fechadas e aquecidas são utilizadas (Breaux \& Miller, 1987).

Rao (1980) estudou a germinação de cariopses oriundas de quatro parentais em dois tipos de cruzamentos, autofecundação e biparentais, em Barbados. Após manter as cariopses em toalhas de papel umedecidas por quatro dias em germinador, obteve, em média, entre 3,1 e 22,7\% de germinação.

Silva (1977) recomendou como condições ótimas para o teste de germinação de cariopses de cana-de-açúcar, a temperatura de $30^{\circ} \mathrm{C}, \mathrm{pH}$ do substrato de 7,0 , luz artificial, por meio de quatro lâmpadas fluorescentes de $20 \mathrm{~W}$ cada, e umidade relativa do ar entre 75 e $90 \%$. Cesnik \& Miocque (2004) afirmaram que o controle da temperatura é muito importante para o sucesso da germinação de cariopses de cana-de-açúcar, sendo a temperatura ideal situada entre 25 e $32^{\circ} \mathrm{C}$, a crítica ao redor dos $18,5^{\circ} \mathrm{C}$, e que abaixo disso a germinação é inibida.

O programa de melhoramento de cultivares de canade-açúcar do Instituto Agronômico de Campinas (IAC) realiza seus cruzamentos no Sul do estado da Bahia, na Estação Experimental de Camamu. Após a obtenção, as sementes são enviadas para Jaú, estado de São Paulo. Entretanto, o processo de germinação é realizado em casa de vegetação, com condições não controladas. Assim, a finalidade desse trabalho foi comparar os ambientes para a germinação das cariopses e a produção de plântulas do Programa Cana IAC.

Dois experimentos foram desenvolvidos em delineamento experimental de blocos casualizados com quatro repetições de 100 sementes, na Unidade de Pesquisa e Desenvolvimento de Jaú (SP), da Agência Paulista de Tecnologia dos Agronegócios (APTA) Regional CentroOeste. Um deles foi montado sob condições de laboratório, em câmara de germinação e outro em casa de vegetação, em condições não controladas, sendo cada tratamento constituído por cariopses provenientes de seis cruzamentos (IACSP94-3581 x SP00-2415, IACSP97-3376 x SP96-1019, IACSP94-5041 x SP93-1213, IACSP97-2109 x SP96-1414, IACSP97-3313 x SP96-1414 e IACSP97-2120 x IAC87-3396). Conduziu-se o experimento de 30/09 a 09/10/2004, isso é, até o $10^{\circ}$ dia após a semeadura, quando a germinação das cariopses se estabilizou. Esse período para germinação leva em consideração a experiência da equipe do Centro de Cana do IAC na produção de plântulas de cana-de-açúcar para fins de Melhoramento Genético.

Os cruzamentos genéticos foram escolhidos de acordo com a porcentagem de germinação de suas cariopses, obtida previamente pela rotina da Estação Experimental de Camamu (BA), pertencente ao Centro de Tecnologia Canavieira (CTC). Esse teste de germinação é feito com $0,5 \mathrm{~g}$ de cariopses distribuídos em caixas plásticas transparentes com tampa, de 11,5 x 11,5 x 3,5 cm (gerbox), com solução de Agar, na concentração de $8 \mathrm{~g} \mathrm{~L}^{-1}$ de água. As caixas são devidamente lacradas e colocadas em germinador, com temperatura controlada de $30^{\circ} \mathrm{C}$ e com iluminação de luz fria por $24 \mathrm{~h}$. A avaliação da germinação é feita após quatro dias, com a contagem das plântulas. Com a obtenção das porcentagens de germinação, foram escolhidos os seis cruzamentos, sendo três com baixa e três com média/alta porcentagem de germinação (Tabela 1).

Tabela 1 - Resultado do teste de germinação prévio realizado na Estação Experimental de Camamu (BA).

\begin{tabular}{cc}
\hline Famílias & $\begin{array}{c}\text { Sementes germinadas } \\
\text { em } 0,5 \mathrm{~g}^{(1)}\end{array}$ \\
\hline IACSP94-3581 x SP00-2415 & 10 \\
IACSP97-3376 x SP96-1019 & 6 \\
IACSP94-5041 x SP93-1213 & 700 \\
IACSP97-2109 x SP96-1414 & 100 \\
IACSP97-3313 x SP96-1414 & 400 \\
IACSP97-2120 x IAC87-3396 & 30 \\
\hline
\end{tabular}

${ }^{(1)}$ Método Centro de Tecnologia Canavieira. 
Em Jaú (SP), os testes de germinação nos dois ambientes foram conduzidos utilizando-se como substrato, a vermiculita de granulometria fina. O substrato foi esterilizado em estufa a $200^{\circ} \mathrm{C}$, por um período de quatro horas, segundo Brasil (1992), e posteriormente foi acondicionado em caixas de plástico de $32 \times 24 \times 7,0 \mathrm{~cm}$, sendo as caixas preenchidas até $6,5 \mathrm{~cm}$ de altura.

Após a semeadura feita em substrato umedecido com $300 \mathrm{~mL}$ de água, as caixas foram levadas a cada um dos ambientes para a instalação dos experimentos. Aquelas colocadas na câmara de germinação foram mantidas à temperatura constante de $32^{\circ} \mathrm{C}$, umidade relativa entre 51 e $99 \%$ e fotoperíodo de 12 horas obtido com quatro lâmpadas de luz branca de $20 \mathrm{~W}$, cada uma perfazendo 50 $\mu \mathrm{mol} \mathrm{m} \mathrm{m}^{-2} \mathrm{~s}^{-1}$ de irradiância. Na casa de vegetação, as temperaturas e umidades relativas do período foram registradas diariamente (Tabela 2). A cada três dias, as caixas foram avaliadas quanto à massa para reposição do volume de água correspondente ao valor inicial de cada parcela experimental.

Foram realizadas avaliações do número de plântulas emergidas, em intervalos de 24 horas, após a instalação do teste, sendo o índice de velocidade de germinação (IVG), contando-se todas as plântulas de acordo com Maguire (1962), a porcentagem e os dias para emergência, conforme Edmond \& Drapala (1958). A massa de 100 sementes de cada cruzamento também foi avaliada, com o propósito de verificar-se sua influência nesses atributos. Essa massa foi determinada por meio de quatro repetições em balança eletrônica de precisão Gehaka, com capacidade de 2000 g e legibilidade de duas casas decimais. $\mathrm{O}$ teor de umidade das cariopses foi considerado semelhante e em equilíbrio com o ambiente, após cinco minutos da retirada das embalagens e durante o manuseio.

Primeiramente, os experimentos foram analisados separadamente. Como os quadrados médios do resíduo destes foram semelhantes para os atributos avaliados, realizou-se a análise conjunta dos dois experimentos. Portanto, os dados foram submetidos à análise de variância e comparação de médias feitas pelo teste de Tukey, a 5\% de significância. Devido a resultados com valor zero, todos os dados foram transformados pela expressão matemática $\sqrt{x+1,0}$, antes da análise. As médias utilizadas nas tabelas são as originais; no entanto, a comparação entre elas está relacionada com a análise estatística dos resultados transformados.

Os resultados da Tabela 3 indicam diferenças entre os cruzamentos, quanto à massa de 100 cariopses, permitindo destacar os cruzamentos IACSP94-5041 x SP931213 e IACSP97-2109 x SP96-1414 como os produtores de cariopses com as maiores massas; a IACSP97-2120 x IAC87-3396 como o de menor, e a IACSP97-3376 x SP961019 como intermediário. Esses resultados sugerem diferenças no potencial fisiológico das cariopses e na qualidade da muda a ser produzida, conforme já observado por Carvalho \& Nakagawa (2000). Para ambientes de germinação não foram encontradas diferenças, significando que as massas de 100 cariopses de cada cruzamento destinado, para cada ambiente foram similares.

O IVG representa o número de plântulas emergidas por dia; portanto, quanto maior o seu valor, maior a velocidade de germinação e maior o vigor. Nesse sentido, o maior IVG foi obtido para a cruzamento IACSP94-5041 x SP93-1213 e os menores para os cruzamentos IACSP943581 x SP00-2415, IACSP97-3376 x SP96-1019 e IACSP972120 x IAC87-3396. Quanto aos ambientes, verificou-se que a câmara de germinação favorece a manifestação desse atributo, proporcionando maior IVG aos lotes cariopses, provavelmente devido às condições terem sido mais uniformes, visto que na casa de vegetação, por não haver controle das condições de trabalho, houve grandes amplitudes térmicas e de umidade relativa do ar (Tabela 2), não seguindo às recomendações de Silva (1977) de que o ambiente para germinação deve ter poucas oscilações de temperatura e teor de umidade. Houve interação significativa entre cruzamento e ambiente.

Tabela 2 - Temperaturas e umidades relativas máximas e mínimas no interior da casa de vegetação, no período de 30/09 a $09 / 10 / 2004$.

\begin{tabular}{llllllllllll}
\hline \multirow{2}{*}{ Condições } & & \multicolumn{10}{c}{ Dias após a semeadura } \\
\cline { 3 - 14 } T $\left({ }^{\circ} \mathrm{C}\right)$ & Max. & 43,9 & 41,9 & 35,9 & 32,5 & 30,3 & 22,8 & 35,9 & 37,4 & 37,4 & 36,3 \\
\hline \multirow{2}{*}{ UR $(\%)$} & Min. & 20,1 & 19,9 & 17,0 & 15,9 & 17,9 & 17,9 & 17,1 & 15,4 & 14,3 & 10,0 \\
\hline & Max. & 71 & 65 & 69 & 57 & 85 & 96 & 99 & 85 & 85 & 97 \\
\cline { 2 - 12 } & Min. & 34 & 20 & 20 & 43 & 42 & 51 & 47 & 44 & 41 & 42 \\
\hline
\end{tabular}

Legenda: T: temperatura em graus centígrados e UR: umidade relativa do ar, em porcentagem. 
Tabela 3 - Massa de 100 cariopses (M100), índice de velocidade de germinação (IVG), dias para emergência (DE) e porcentagem de germinação $(\mathrm{G})$ de cariopses de seis cruzamentos de cana-de-açúcar, submetidas a dois ambientes.

\begin{tabular}{llcccc}
\hline \multirow{2}{*}{ Tratamentos } & P100 & IVG & DE & G \\
\cline { 2 - 5 } & & $\mathrm{G}$ & plantas dia $^{-1}$ & Dias & $\%$ \\
\hline \multirow{5}{*}{ Cruzamentos } & IACSP94-3581 x SP00-2415 & $0,036 \mathrm{c}^{(1)}$ & $0,051 \mathrm{~d}$ & 3,625 & $0,500 \mathrm{~d}$ \\
& IACSP97-3376 x SP96-1019 & $0,048 \mathrm{bc}$ & $0,155 \mathrm{~d}$ & 4,250 & $0,750 \mathrm{~d}$ \\
& IACSP94-5041 x SP93-1213 & $0,066 \mathrm{a}$ & $8,966 \mathrm{a}$ & 4,723 & $38,875 \mathrm{a}$ \\
& IACSP97-2109 x SP96-1414 & $0,055 \mathrm{ab}$ & $2,740 \mathrm{c}$ & 4,939 & $12,625 \mathrm{c}$ \\
& IACSP97-3313 x SP96-1414 & $0,038 \mathrm{c}$ & $6,062 \mathrm{~b}$ & 4,166 & $22,625 \mathrm{~b}$ \\
& IACSP97-2120 x IAC87-3396 & $0,015 \mathrm{~d}$ & $0,342 \mathrm{~d}$ & 5,906 & $1,625 \mathrm{~d}$ \\
\hline \multirow{2}{*}{ Ambientes } & Câmara de Germinação & 0,045 & $2,848 \mathrm{a}$ & $2,735 \mathrm{~b}$ & 8,759 \\
& Casa de Vegetação & 0,041 & $1,782 \mathrm{~b}$ & $5,884 \mathrm{a}$ & 9,065 \\
\hline \multirow{3}{*}{ Valor de F } & $29,89^{* *}$ & $130,49 * *$ & $2,53^{\text {ns }}$ & $346,81^{* *}$ \\
& Cruzamentos (C) & $1,58^{\mathrm{ns}}$ & $21,73^{* *}$ & $27,55^{* *}$ & $0,27^{\mathrm{ns}}$ \\
& Ambientes de Germinação (A) & $0,96^{\mathrm{ns}}$ & $11,40^{* *}$ & $2,60{ }^{\mathrm{ns}}$ & $6,76^{*}$ \\
\hline & C x A & 0,43 & 12,3 & 20,02 & 10,26 \\
\hline
\end{tabular}

${ }^{1}$ Médias originais, análise de variância feita com médias transformadas $\sqrt{x+1,0}$. Médias seguidas de mesma letra dentro de cada atributo (cruzamentos e ambientes) não diferem estatisticamente, a 0,05 de probabilidade, pelo teste de Tukey.

ns, *, ** não significativo, significativo a 0,05 e a 0,01 respectivamente.

Na Tabela 4 está apresentado o desdobramento da interação cruzamentos $\mathrm{x}$ ambientes de germinação para $\mathrm{o}$ atributo índice de velocidade de germinação. Houve diferenças entre cruzamentos nos dois ambientes avaliados; entretanto, os dois cruzamentos com maiores taxas de germinação pelo teste do CTC exibiram comportamentos distintos entre os ambientes. O cruzamento IACSP94-5041 x SP93-1213, escolhido por apresentar a maior germinação (Tabela 1), mostrou no ensaio o maior IVG na câmara de germinação com condições controladas, seguido do cruzamento IACSP97-3313 x SP961414 , com valor de 6,89 plantas dia ${ }^{-1}$, mas diferenciando-se daquele significativamente. Por outro lado, nas condições não controladas da casa de vegetação, o maior valor para o IVG foi obtido por esse último cruzamento, igualando-se significativamente com o IACSP94-5041 x SP93-1213 com um IVG de 4,87 plantas dia ${ }^{-1}$. Esses resultados revelam que a câmara de germinação potencializou apenas a germinação das cariopses do cruzamento IACSP94-5041 x SP93-1213, que além de diferenciar significativamente do resultado obtido na casa de vegetação, ainda mostrou um acréscimo no IVG de $268 \%$.

Para a DE nenhuma diferença significativa foi encontrada entre cruzamentos (Tabela 3), mas deve analisar-se esse resultado com ressalvas, pois o coeficiente de variação apresentou-se um tanto quanto elevado. Como DE estimam o tempo médio de germinação usado para avaliar a emergência da plântula (Ranal \& Santana, 2006), tem-se que estatisticamente esses valores equivaleram-se, não indicando um cruzamento com maior vigor. Entretanto, houve diferenças entre ambientes, em que a casa de vegetação exibiu maior valor, portanto, maior tempo médio gasto para germinar. Não houve interação entre cruzamentos e ambiente de germinação.

Diferenças significativas foram encontradas entre cruzamentos para porcentagem de germinação (G) ao final de 10 dias (Tabela 3), em que o cruzamento IACSP94-5041 x SP931213 apresentou o maior valor, enquanto que os cruzamentos IACSP94-3581 x SP00-2415, IACSP97-3376 x SP96-1019 e IACSP97-2120 x IAC87-3396 apresentaram os menores valores. Apesar das diferenças obtidas, nota-se a baixa porcentagem de germinação dos lotes, mesmo no cruzamento de maior valor. Quanto aos ambientes, não foi obtida diferença significativa entre a câmara de germinação e a casa de vegetação, indicando que, ao final do período necessário para a germinação, os resultados dos dois ambientes se equivaleram. Portanto, nesse atributo, as maiores amplitudes de temperatura e umidade relativa não prejudicaram o resultado final de obtenção de plântulas, apesar da interação significativa entre cruzamentos e ambientes.

$O$ desdobramento da interação entre cruzamento $\mathrm{x}$ ambiente de germinação, para o atributo porcentagem de 
germinação, está apresentado na Tabela 5. Entre cruzamentos, tanto na câmara de germinação quanto na casa de vegetação, os valores corresponderam, proporcionalmente, aos obtidos previamente pelo CTC. $\mathrm{Na}$ câmara de germinação, os valores ficaram entre $0,00 \mathrm{e}$ $44,25 \%$ e na casa de vegetação entre 0,75 e $33,50 \%$. Essa ampla variação entre cruzamentos também foi observada por Dutt et al. (1938) e Rao (1980) que registraram valores entre 3,1 e $22,7 \%$ de germinação. As cariopses do cruzamento IACSP94-5041 x SP93-1213 tiveram maior porcentagem de germinação na câmara de germinação do que na casa de vegetação, levando a inferir que as condições controladas de temperatura e umidade proporcionaram essa maior porcentagem. Por outro lado, o cruzamento IACSP97-2109 x SP96-1414 apresentou maior porcentagem de germinação na casa de vegetação, diferenciando-se da obtida na câmara de germinação, apesar das condições não controladas do primeiro ambiente. No entanto, esse fato ainda manteve a germinação das cariopses desse cruzamento dentro do posicionamento obtido no teste realizado pelo CTC (Tabela 1). Dutt et al.
(1938) observaram que cariopses provenientes de cruzamentos de Saccharum officinarum x $S$. spontaneum e de cruzamentos entre híbridos apresentaram porcentagens de germinação variadas conforme o ano do teste, como consequência das condições climáticas, uma vez que não dispunham de condições controladas. Concluíram que lotes de cariopses provenientes de cruzamentos diferentes apresentavam desempenhos distintos com a variação climática.

Portanto, apesar da câmara de germinação proporcionar condições controladas ao ambiente, a casa de vegetação do Programa Cana IAC, que possui condições semicontroladas, ofereceu no ano de 2004 ambiente favorável para produção de plântulas e pode continuar sendo utilizada para semeadura de cariópses para o programa de melhoramento genético de cana-de-açúcar, entretanto estudos em diferentes anos devem ser realizados para um melhor conhecimento, em variadas condições climáticas. As condições controladas da câmara de germinação favoreceram o IVG e a VG das cariopses de cana-de-açúcar estudadas.

Tabela 4 - Índice de velocidade de germinação de cariopses de seis cruzamentos de cana-de-açúcar (plantas dia-1), em dois ambientes ${ }^{1}$.

\begin{tabular}{ccc}
\hline \multirow{2}{*}{ Cruzamentos } & \multicolumn{2}{c}{ Ambientes de Germinação } \\
\cline { 2 - 3 } & Câmara de Germinação & Casa de Vegetação \\
\hline IACSP94-3581 x SP00-2415 & $0,00 \mathrm{dA}$ & $0,10 \mathrm{cA}$ \\
IACSP97-3376 x SP96-1019 & $0,21 \mathrm{dA}$ & $0,10 \mathrm{cA}$ \\
IACSP94-5041 x SP93-1213 & $13,06 \mathrm{aA}$ & $4,87 \mathrm{aB}$ \\
IACSP97-2109 x SP96-1414 & $2,73 \mathrm{cA}$ & $2,75 \mathrm{bA}$ \\
IACSP97-3313 x SP96-1414 & $6,89 \mathrm{bA}$ & $5,23 \mathrm{aA}$ \\
IACSP97-2120 x IAC87-3396 & $0,52 \mathrm{dA}$ & $0,17 \mathrm{cA}$ \\
\hline
\end{tabular}

${ }^{1}$ Médias seguidas de letras diferentes, minúsculas na coluna e maiúsculas na linha, diferem estatisticamente, a 0,05\% de probabilidade pelo teste de Tukey.

Tabela 5 - Germinação de sementes de seis famílias de cana-de-açúcar (G\%), em dois ambientes

\begin{tabular}{ccc}
\hline \multirow{2}{*}{ Cruzamentos } & \multicolumn{2}{c}{ Ambientes de Germinação } \\
\cline { 2 - 3 } & Câmara de Germinação & Casa de Vegetação \\
\hline IACSP94-3581 x SP00-2415 & $0,00 \mathrm{dA}$ & $1,00 \mathrm{dA}$ \\
IACSP97-3376 x SP96-1019 & $0,75 \mathrm{dA}$ & $0,75 \mathrm{dA}$ \\
IACSP94-5041 x SP93-1213 & $44,25 \mathrm{aA}$ & $33,50 \mathrm{aB}$ \\
IACSP97-2109 x SP96-1414 & $9,25 \mathrm{cB}$ & $16,00 \mathrm{cA}$ \\
IACSP97-3313 x SP96-1414 & $22,50 \mathrm{bA}$ & $22,75 \mathrm{bA}$ \\
IACSP97-2120 x IAC87-3396 & $2,00 \mathrm{dA}$ & $1,25 \mathrm{dA}$ \\
\hline
\end{tabular}

${ }^{1}$ Médias seguidas de letras diferentes, minúsculas na coluna e maiúsculas na linha, diferem estatisticamente, a 5\% de probabilidade pelo teste de Tukey.

Ciênc. agrotec., Lavras, v. 34, Edição Especial, p. 1604-1609, 2010 


\section{REFERÊNCIAS BIBLIOGRÁFICAS}

BRASIL. Ministério da Agricultura e Reforma Agrária. Regras para análise de sementes. Brasília: SNDA/ DNDV/CLAV, 1992. 365p.

BREAUX, R.D.; MILLER, J.D. Seed handling, germination and seedling propagation. In: HEINZ, D.J. (Ed.). Sugarcane improvement through breeding. Elsevier: Amsterdam, 1987. p.385-407.

CARVALHO, N.M.; NAKAGAWA, J. Sementes: ciência, tecnologia e produção. 4.ed. Campinas: Fundação Cargill, 2000. 588p.

CESNIK, R.; MIOCQUE, J.J.Y. Melhoramento da cana-deaçúcar. Brasília: Embrapa, 2004. 307p.

DILLEWIJN, C. van Botany of sugarcane. Walthan: Chronica Botanica, 1952.371p.

DUTT, N.L.; KRISHNASWAMI, M.K.; SUBBA RAO, K.S. A note on seed-setting and seed-germination in certain sugarcanes. The Indian Journal of Agricultural Sciences, New Delhi, v.8, n.4, p.429-433, 1938.

EDMOND, J.B.; DRAPALA, W.J. The effects of temperature, sand and soil, and acetone on germination of okra seeds. Proceedings of the American Society Horticultural Science, Alexandria, v.71, p.428-434, 1958.

HEINZ, D.J. Temperature effect on fuzz (true seed) germination. Honolulu: Hawaii Sugarcane Planters Association, 1975. 7p. (Annual report).
LUCCHESI, A.A. Cana-de-açúcar (Saccharum spp.). In: CASTRO, P.C.; KLUGE, R.A. (Coord.). Ecofisiologia de culturas extrativas: cana-de-açúcar; seringueira; coqueiro; dendezeiro e oliveira. Cosmópolis: Stoller do Brasil, 2001. p.13-45.

MACHADO JÚNIOR, G.R. Melhoramento da cana-deaçúcar. In: PARANHOS, S.B. (Coord.). Cana-de-açúcar: cultivo e utilização. Campinas: Fundação Cargill, 1987. v.1, p.165-186.

MAGUIRE, J.D. Speed of germination-aid in selection and evaluation for seedling emergence and vigor. Crop Science, Madison, v.2, n.1, p.176-177, 1962.

MIOCQUE, J.J.Y.; MACHADO JÚNIOR, G.R. Review of the sugarcane varieties and breeding in Brazil. Sugar Journal, New Orleans, v.40, n.7, p.9-13, 1977.

RANAL, M.A.; SANTANA, D.G. How and why to measure the germination process? Revista Brasileira de Botânica, São Paulo, v.29, n.1, p.1-11, 2006.

RAO, P.S. Fertility, seed storage, and seed viability in sugarcane. In: INTERNATONAL SOCIETY SUGARCANE CANE TECHNOLOGISTS CONGRESS, 17., 1980, Manila. Proceedings... Manila: ISSCT, 1980. p.1236-1240.

SILVA, W.M. Production of sugarcane seedlings by the method of fuzz processing and early transplantation. In: INTERNATONAL SOCIETY OF SUGARCANE TECHNOLOGISTS CONGRESS, 16., 1977, São Paulo. Proceedings... São Paulo: ISSCT, 1977. p.918-924. 